\title{
Wireality: Enabling Complex Tangible Geometries in Virtual Reality with Worn Multi-String Haptics
}

\author{
Cathy Fang Yang Zhang Matthew Dworman Chris Harrison \\ Carnegie Mellon University, Pittsburgh, PA, USA \\ cathyfang@cmu.edu,\{mdworman,yang.zhang,chris.harrison\}@cs.cmu.edu
}

\begin{abstract}
Today's virtual reality (VR) systems allow users to explore immersive new worlds and experiences through sight. Unfortunately, most VR systems lack haptic feedback, and even high-end consumer systems use only basic vibration motors. This clearly precludes realistic physical interactions with virtual objects. Larger obstacles, such as walls, railings, and furniture are not simulated at all. In response, we developed Wireality, a self-contained worn system that allows for individual joints on the hands to be accurately arrested in 3D space through the use of retractable wires that can be programmatically locked. This allows for convincing tangible interactions with complex geometries, such as wrapping fingers around a railing. Our approach is lightweight, lowcost, and low-power, criteria important for future, worn consumer uses. In our studies, we further show that our system is fast-acting, spatially-accurate, high-strength, comfortable, and immersive.
\end{abstract}

\section{Author Keywords}

Virtual Reality; Haptics; Force Feedback; String-Driven; Touch; Grasp.

\section{CSS Concepts}

Human-centered computing $\rightarrow$ Human computer interaction $(\mathrm{HCI}) \rightarrow$ Interaction devices $\rightarrow$ Haptic devices.

\section{INTRODUCTION}

Virtual reality (VR) systems, such as the Oculus Quest [15] and HTC Vive [28], use controllers for tracking the hands, capturing buttoned input, and delivering basic vibrotactile haptic feedback. The latter is insufficient to produce immersive physical interactions with virtual objects. More critically, large obstacles like walls, railings, and furniture key elements in most VR worlds - are not simulated at all. The current state-of-the-art in consumer VR systems is a vibration alert when a hand intersects a virtual object or obstacle - falling far short of any reality.

Permission to make digital or hard copies of part or all of this work for personal or classroom use is granted without fee provided that copies are not made or distributed for profit or commercial advantage and that copies bear this notice and the full citation on the first page. Copyrights for third-party components of this work must be honored. For all other uses, contact the Owner/Author.

CHI '20, April 25-30, 2020, Honolulu, HI, USA.

(C) 2020 Copyright is held by the owner/author(s).

ACM ISBN 978-1-4503-6708-0/20/04.

DOI: https://dx.doi.org/10.1145/3313831.3376470

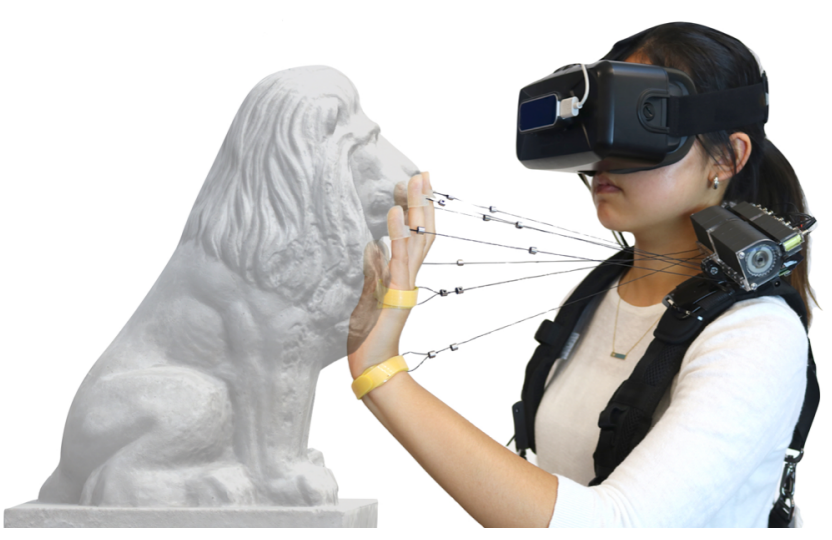

Figure 1. Wireality enables strong, whole-hand haptic feedback for complex objects in VR experiences.

This shortcoming has long been recognized, and researchers have looked into ways to bring rich haptics to VR experiences for many decades [36]. As we will review in greater detail, most systems have focused on hand haptics, such that virtual objects feel as though they are being held and are able to be moved in space. Less common are systems that attempt to arrest the hands and arms to simulate immovable objects, such as walls. To achieve this effect, systems often use mechanical exoskeletons $[10,23,57]$ or fixed infrastructure in the environment $[29,40,54]$, neither of which is particularly practical for consumer use.

We set out to design a new VR haptic system that was entirely self-contained and mobile. This implied a worn system, which in turn, meant our approach needed to be both lightweight and battery-powered. To simulate interactions with heavy or fixed objects, we needed a system that was both fast-acting and able to provide large arresting forces. Finally, in order to be a plausible consumer accessory, it should cost no more than $\$ 50$ in volume production.

In this paper, we present our work on Wireality, which meets the above design criteria. Our system is comprised of modular, spring-loaded cables, which we can programmatically lock with a ratchet gear and a solenoid-driven pawl (Figure 1). This locking action takes under $30 \mathrm{~ms}$, provides up to $180 \mathrm{~N}$ of arresting force, and yet only consumes $0.024 \mathrm{mWh}$ of energy (allowing our approach to be battery powered and mobile). Each module is responsible for limiting one degree of freedom on the hand. With many modules acting together as a unit, Wireality enables interactions with 
complex geometries as users reach out towards, e.g., virtual walls, railings, and other objects (Figure 1). Our prototype weighs $273 \mathrm{~g}$ in total, though only $11 \mathrm{~g}$ is worn on the hands (making it considerably lighter than, e.g., an HTC Vive controller at 203g).

We first review related research, including several key papers that served as inspiration. We then discuss our iterative development of Wireality and the final proof-of-concept implementation we created. Next, we discuss inherent limitations of our approach, along with example uses where it excels. We conclude with a two-part evaluation, one quantitative and the other qualitatively oriented.

\section{RELATED WORK}

There has been a significant body of research on haptic feedback on users' heads (e.g., [21, 39, 41]), bodies (e.g., $[12,30,34]$ ), and limbs (e.g., [22, 43, 48]). Closer to this work are VR haptic systems for the arms and hands, which we review in greater detail. We then discuss research that more closely aligns with our technical approach of using string-based mechanisms, concluding with the most closely related systems.

\section{Hand-Centric Haptics in VR}

Contemporary consumer VR products use hand controllers with vibration motors to provide haptic feedback $[15,28]$. However, this approach does not allow free-hand interactions with virtual objects as users' hands are occupied with the controller. In response, researchers have considered form factors that allow for more free-hand movement, such as a squeezable controller [33], a cane [56], and gloves [11, 52]. It is also possible to simulate the texture of virtual objects $[2,53]$ and even gross contour using actuated pin arrays [5], solenoids [7], propellers [24, 26], and electrical muscle stimulation [35].

There is also a large literature on rendering haptic illusions for grasp. For example, Wolverine [8] and Grabity [9] use braking sliders on rails to simulate realistic gripping of rigid objects. PuPoP [50] uses in-hand pneumatics to simulate shapes, while $\mathrm{PaCaPa}$ [47] uses servo motors to control the angle between two wings of a hand-held token. Finally, innumerable force-feedback exoskeletons and computercontrolled mechanical linkages have been developed [10, $23,46,57]$.

\section{String-Based Haptics}

Strings offer a lightweight approach to deliver force feedback, allowing the main mechanism to be placed somewhere less cumbersome. For example, CSHI [54] and Yang et al. [55] used strings to implement a haptic hand grip on a planar surface. HapticPen [16] used three motor-controlled strings running from the user's body to the tip of a pen to simulate haptics for a virtual 2D plane. Similarly, billiARds [42] used two motor-controlled strings connected to a billiard cue tip to provide force feedback. More recently, InSight [20] developed a force feedback system using three motor-controlled strings connected with a handle to im- prove navigation for people with visual impairment. Please also see [19] for a more extensive review.

\section{On-Hand String Haptics}

Prior work has also attached strings directly to users' hands, which is similar to our system. SPIDAR [29] connected a user's finger to four corners of a large cubic frame via motor-controlled strings and could provide force feedback in any direction. Similarly, Hou et al. [27] used strings to create a haptic touchscreen. Most relevant to our work are string-driven systems that are self-contained and wearable. The WireMan project [6] developed one-string [4] and three-string worn systems [3, 38] that could arrest a user's index finger in 3D space. Tsai et al. [51] built a wrist-worn system with controllable elastics that could simulate solid or compliant objects. Other string-like mechanisms have employed electrostatic brakes [25], fluidics [31], and pneumatic actuators $[44,45]$ to constrain users' joints and finger movement.

Our system moves beyond this prior work with a truly wearable and mobile implementation, which delivers haptic feedback to the whole arm and each hand joint independently. Additionally, previous string-based systems have used motors to maintain string tension, whereas Wireality leverages steel springs, which are low-cost, lightweight, and require significantly less power with the locking mechanism we designed.

\section{WIREALITY IMPLEMENTATION}

We wished to design a modular, wearable system that enabled tangible hand interactions with complex virtual objects. In order to be worn, our system needed to be lightweight and energy-efficient. We also aimed for a design that could be mass-produced for under $\$ 50$. We now describe our implementation, along with notable insights found during iterative development.

\section{String Material}

One of our first design considerations was choosing an appropriate string material for arresting hand movements. We tested many strings, with varying max pulling force, durability, and flexibility (e.g., fishing wire and nylon). We ultimately selected nylon-coated braided steel cables, which are strong and thin, and can easily wrap around a small diameter reel.

\section{String Retraction}

Any slack in strings means the system cannot accurately arrest a user's hand and could even lead to tangles. Initially, we built motor-based retracting mechanisms, which would also afford more dynamic haptic effects (Figure 2). However, in order to resist the force of a user's arm, we resorted to using increasingly heavy, bulky, and power-consumptive motors. In some of our larger designs, we even started to face thermal dissipation issues.

Instead, we pivoted and turned to flat torsional springs, commonly found in retracting badges, though we use commercial retractor modules [17] in our final design (Figure 


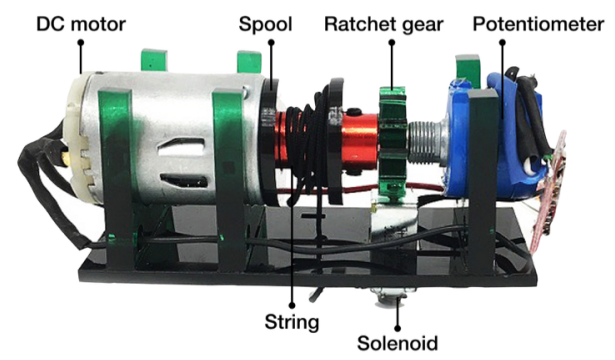

Figure 2. An earlier prototype using a motor for string retraction and a potentiometer for tracking hand positions.

3). Although torsional springs only offer a fixed pull force (i.e., determined by the spring constant), we found an $80 \mathrm{~g}$ pull force retractor to be suitable for removing slack at typical human movement speeds, while not being overly distracting. Most importantly, steel torsional springs are inexpensive, lightweight, compact, and robust.

\section{Arresting Mechanism}

Low latency is crucial in accurately arresting a user's hand in creating a convincing haptic experience. Most previous related systems used stepper motors to retract and lock strings, but as noted previously, these are bulky and energyconsumptive. For both our motor-based and spring-based prototypes (Figures 2 and 3), we designed a locking, ratchet-and-pawl mechanism, which is lightweight, compact, and consumes no power once engaged. We drew inspiration from locking mechanisms used in seat belts and semiautonomous belays, as well as from recent HCI work, Wolverine [8] in particular.

Since the pawl can only latch the ratchet gear at discrete points (tooth boundaries), the resolution of the locking mechanism increases as the number of teeth grows. However, it is important to consider the pressure angle, pitch depth, and the pitch diameter of the ratchet gear, as they influence the size and precision of the mechanism. For example, with increased tooth count, the pitch depth would decrease, making it harder for the pawl to engage with the ratchet securely. Alternatively, one can reduce the diameter of the string spool to increase the resolution of the gear.

Our final arresting mechanism design consists of a laser-cut acrylic ratchet gear with a resolution of $8^{\circ}$ per tooth, and it is attached concentrically to our aforementioned commercial retractor [17], which contains a torsional spring, spool, and string. Because the string is wound around a spool with a smaller diameter than the ratchet gear, each tooth equates to is $0.84 \mathrm{~mm}$ of "string travel". We attach a laser-cut acrylic pawl to the armature of a commodity $12 \mathrm{~V}$ DC push-pull solenoid [13] (Figure 3).

When a user's hand collides with a virtual object, our software triggers the solenoid to actuate, pushing the pawl into the ratchet, which locks the spool from further rotation, arresting and ultimately the hand. Force exerted by the user on the string keeps the ratchet latched, and thus the solenoid can be unpowered after a brief interval. Only when users

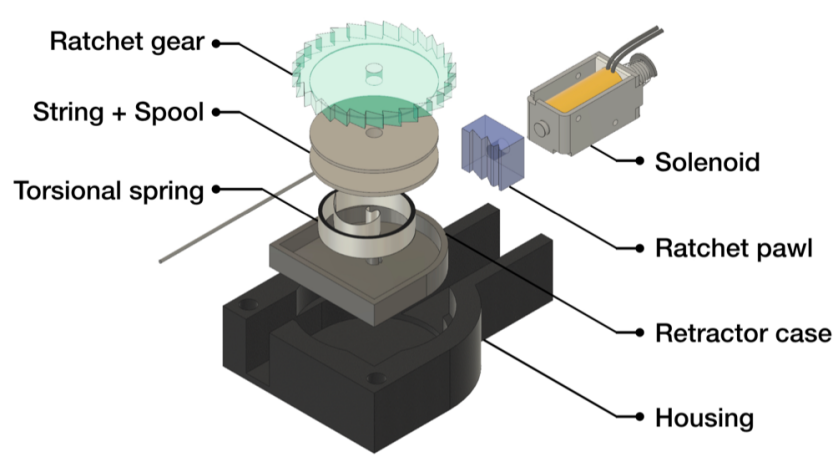

Figure 3. Exploded illustration of one haptic module.

withdraw their hand is the pawl released and the spring can retract any string slack (Figure 4).

\section{Module Housing \& String Guide}

Each locking and retracting mechanism is housed in a custom enclosure 3D-printed using Onyx filament [37], a highstrength and lightweight thermoplastic made from nylon and chopped carbon fiber. The modules can be connected with long bolts (a seven-module unit can be seen in Figure 5). We also included a small laser cut guide at the module aperture to ensure the string would wind on the spool correctly, even if the string departed the module at an oblique angle. Overall dimensions of our final module design measure $4.1 \times 7 \times 1.4 \mathrm{~cm}^{3}$.

\section{Driver Electronics}

To control our solenoid-driven pawls, we used dual HBridge L298N motor drivers [1] controlled by a Teensy 3.2 microcontroller board [49], which receives commands over USB from our VR software (described later). These electronic components are compact and consume practically no power when solenoids are not actuated. In the future, a simple transistor could be used to further reduce cost and size. When the teensy is instructed to lock one of the strings, it actuates the corresponding solenoid for $40 \mathrm{~ms}$, which we found to be the shortest interval for reliable locking.

\section{On-Body Placement}

We explored different placements of Wireality on the body, aiming to give users maximum freedom of movement while not violating the inherent "line of sight" requirement of our strings. We also had to select a comfortable and stable

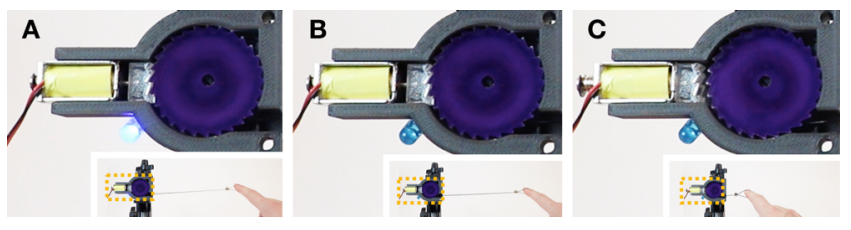

Figure 4. Locking mechanism. Our driver board actuates the solenoid pawl for $\mathbf{4 0}$ milliseconds, which locks the ratchet gear, stopping a joint from further forward movement (A). String tension keeps the gear latched even after the solenoid is not powered (B). When the user pulls back from touching the virtual surface, slack is created in the string, which then releases the ratchet $(C)$. 


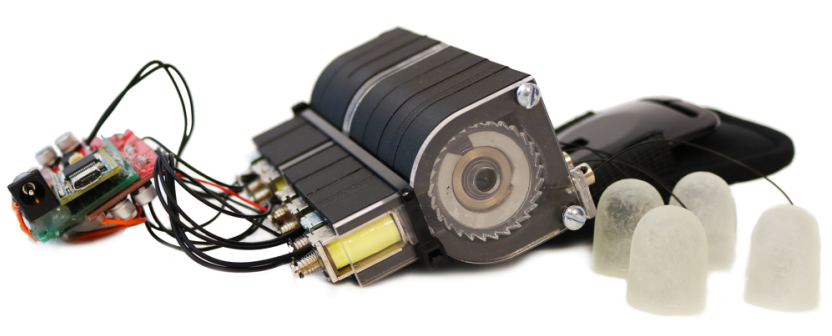

Figure 5. Example Wireality setup with seven haptic modules.

location to wear our Wireality unit, which could be $14 \mathrm{~cm}$ in length for ten modules and experience significant depending on the virtual object. Given the forces exerted by users' hands, it was also important to select an anchor location with the requisite counteracting body mass. Integrating recommendations from Gemperle et al. [18], we ultimately chose the shoulder location and made a vest prototype that allowed participants to easy wear our system (Figure 1).

\section{Hand Attachment Points}

As our device was modular, we were able to experiment with different numbers of hand attachment points. We found that with more points of contact (e.g., tip of a finger, metacarpophalangeal and interphalangeal joints), more complex geometries could be simulated, but at the cost of an increasingly large and heavy device. For instance, allocating 3 strings to each finger, 2 to the thumb, and 1 to the wrist requires 15 modules.

In piloting, we found that such a high degree of instrumentation was not needed to produce realistic haptic effects, and so our final implementation had strings for just the 5 fingertips, 1 for the palm, and 1 for the wrist (i.e., 7 modules). Instead of using gloves, which often make users' hands sweaty and uncomfortable, we 3D-printed finger caps and used Velcro straps for the palm and wrist, which help to distribute the otherwise concentrated pressure. This setup can be seen in Figure 1.

\section{Hand Tracking}

Arresting a user's fingers at points in 3D space to simulate complex virtual objects requires precise tracking of many hand joints. In early prototypes (one example shown in Figure 2), we included a 10-turn potentiometer that allowed us to precisely track a point's distance from the module, but not the azimuth or altitude. Ultimately, we used a Leap Motion [32] attached to the front of a VR headset for hand tracking, which offers 3D locations for all hand joints. To minimize Wireality interfering with the Leap Motion's hand tracking, we used 3D-printed finger caps that appear as the same color as skin in infrared. We also used thin strings that are almost invisible to the camera.

\section{VR Hardware \& Software}

We used an Oculus Rift [14] as a proof-of-concept VR platform. All of our VR scenes were built in Unity. To detect collisions with virtual objects, we assign objectCollider to obstacles and objects in our virtual environments. Upon contact with a joint, an onTriggerEnter event is dis- patched. The event handler sends a locking command to our driver board over USB for the corresponding joint. Collision detection runs in parallel for all finger and hand joints, and the actuation of solenoids does not interfere with one another, allowing for fully independent control, which is essential for supporting complex tangible geometries.

\section{EVALUATION}

We ran a series of empirical tests to characterize the performance of our system. Here we describe our test procedures and results and conclude with comparisons between Wireality and prior work.

\section{Weight}

Weight is a serious consideration for any worn system [18]. Our final module design weighs $30 \mathrm{~g}$; a seven-module unit (including driver electronics and mounts) weighs $273 \mathrm{~g}$ in total. Importantly, most of this mass rests on the shoulder where it is less noticeable and taxing. The collective weight of the elements worn on the hand is a mere $11 \mathrm{~g}$. For comparison, the handheld HTC Vive controller weighs $203 \mathrm{~g}$.

\section{Arresting Force}

Wireality aims to provide sufficient feedback force to simulate heavy objects and obstacles. Simultaneously, our mechanism must be capable of rapidly and repeatedly arresting an entire mass of the arm. To assess this, we performed pull force stress tests on our modules using highspeed cameras and digital force gauges. We found a mean failure force of $186 \mathrm{~N}$.

\section{Solenoid Actuation Time}

To actuate the solenoids in our haptic modules, we experimented with drive voltages ranging from 5 to $12 \mathrm{~V}$, and actuation duration from 10 to $1000 \mathrm{~ms}$. We found $12 \mathrm{~V}$ for $40 \mathrm{~ms}$ offered a good balance between speed and reliable locking. Note that in most cases, the ratchet is completely locked within $25 \mathrm{~ms}$, but there are occasional outliers due to rapid user movement or poor interfacing of the pawl.

\section{Power Consumption}

A truly mobile system needs to be energy efficient, allowing for untethered power sources. Our haptic modules draw $2.19 \mathrm{~W}(183 \mathrm{~mA}$ at $12 \mathrm{~V})$ of power when the solenoid is actuated. As we only actuate solenoids for $40 \mathrm{~ms}$ to achieve locking, each locking event consumes just $0.024 \mathrm{mWh}$ (0.088 Joules). For reference, the battery-powered Oculus Quest headset contains a $14,000 \mathrm{mWh}$ battery or enough energy for over half a million locking events. In other words, we expect that our device would have minimal impact on the battery life of most untethered VR headsets.

\section{Latency}

Minimizing latency is critical in rendering realistic haptic feedback. We measured our system's end-to-end latency specifically, the elapsed time from the moment a user's finger collides with a virtual obstacle in VR to the moment that the user's finger is arrested in physical space. On average, total latency was $29 \mathrm{~ms}$. The largest contributing sources of latency are: Leap Motion hand tracking (9ms), 


\begin{tabular}{|c|c|c|c|c|c|c|c|c|c|c|c|c|}
\hline & Wireality & $\begin{array}{c}\text { VR Haptic } \\
\text { Drones [26] } \\
\end{array}$ & CLAW [10] & $\begin{array}{c}\text { Normal } \\
\text { Touch [5] } \\
\end{array}$ & ElasticVR[51] & Wolverine [8] & DextrES [25] & PuPoP [50] & TORC [33] & $\begin{array}{c}\text { Haptic } \\
\text { Revolver [53] } \\
\end{array}$ & $\begin{array}{c}\text { Thor's } \\
\text { Hammer [24] }\end{array}$ & $\begin{array}{c}\text { Haptic Link } \\
{[46]}\end{array}$ \\
\hline $\begin{array}{l}\text { Sensing } \\
\text { Points }\end{array}$ & whole hand & whole hand & thumb + index & one finger & whole hand & $\begin{array}{l}\text { thumb, index + } \\
\text { middle fingers }\end{array}$ & thumb + index & whole hand & $\begin{array}{l}\text { thumb, index }+ \\
\text { middle fingers }\end{array}$ & one finger & arm & arm \\
\hline $\begin{array}{c}\text { Actuator } \\
\text { Type }\end{array}$ & $\begin{array}{c}\text { ratchet gear } \\
\text { with solenoid } \\
\text { pawl + strings }\end{array}$ & drones & $\begin{array}{l}\text { servo motor }+ \\
\text { voice coil } \\
\text { actuator }\end{array}$ & servo motor & $\begin{array}{l}\text { rotation motor } \\
+ \text { elastic band }\end{array}$ & one-way brake & $\begin{array}{c}\text { electrostatic } \\
\text { brake }+ \\
\text { piezoelectric } \\
\text { actuator }\end{array}$ & pneumatic & $\begin{array}{l}\text { vibrotactile } \\
\text { motor + voice } \\
\text { coil actuator }\end{array}$ & $\begin{array}{c}\mathrm{DC} \text { motor }+ \\
\text { wheel }\end{array}$ & $\begin{array}{c}\text { motor }+ \\
\text { propeller }\end{array}$ & $\begin{array}{c}\text { linear actuator } \\
\text { + ball-and- } \\
\text { socket }\end{array}$ \\
\hline $\begin{array}{c}\text { Haptic } \\
\text { feedback }\end{array}$ & collision & collision & $\begin{array}{c}\text { collision + grip } \\
+ \text { texture }\end{array}$ & $\begin{array}{l}\text { collision + } \\
\text { compliance }\end{array}$ & $\begin{array}{c}\text { impact }+ \\
\text { compliance }\end{array}$ & grip & $\begin{array}{c}\text { grip + } \\
\text { compliance }\end{array}$ & $\begin{array}{c}\text { grip + } \\
\text { compliance }\end{array}$ & $\begin{array}{c}\text { texture }+ \\
\text { compliance }\end{array}$ & texture & tug & bimanual grip \\
\hline $\begin{array}{c}\text { Max. Force } \\
\text { per Finger }\end{array}$ & $183 \mathrm{~N}$ & $\mathrm{n} / \mathrm{a}$ & $30 \mathrm{~N}$ & $\mathrm{n} / \mathrm{a}$ & $14 \mathrm{~N}$ & $106 \mathrm{~N}$ & $20 \mathrm{~N}$ & $\mathrm{n} / \mathrm{a}$ & $\mathrm{n} / \mathrm{a}$ & $3.35 \mathrm{~N}$ & $4 \mathrm{~N}$ & $80 \mathrm{~N}$ \\
\hline $\begin{array}{l}\text { Peak Power } \\
\text { Consumption }\end{array}$ & $2.19 \mathrm{~W}$ & $20.40 \mathrm{~W}$ & $5 \mathrm{~W}$ & $\mathrm{n} / \mathrm{a}$ & $\mathrm{n} / \mathrm{a}$ & $2.89 \mathrm{~W}$ & $<0.12 \mathrm{~W}$ & $24 W$ & $\mathrm{n} / \mathrm{a}$ & $2.50 \mathrm{~W}$ & $204.70 \mathrm{~W}$ & $7.20 \mathrm{~W}$ \\
\hline $\begin{array}{c}\text { Weight on } \\
\text { the Hand }\end{array}$ & $11 \mathrm{~g}$ & $0 \mathrm{~g}$ & $420 \mathrm{~g}$ & $150 \mathrm{~g}$ & $150 \mathrm{~g}$ & $55 \mathrm{~g}$ & $16 \mathrm{~g}$ & $\mathrm{n} / \mathrm{a}$ & $\mathrm{n} / \mathrm{a}$ & $237 \mathrm{~g}$ & $692 \mathrm{~g}$ & $>651 \mathrm{~g}$ \\
\hline Cost & $\$ 35$ & $\$ 30$ & $>\$ 150$ & $\mathrm{n} / \mathrm{a}$ & $\mathrm{n} / \mathrm{a}$ & $\$ 40$ & $\mathrm{n} / \mathrm{a}$ & $\mathrm{n} / \mathrm{a}$ & $<\$ 90$ & $\mathrm{n} / \mathrm{a}$ & $\mathrm{n} / \mathrm{a}$ & $\mathrm{n} / \mathrm{a}$ \\
\hline
\end{tabular}

Table 1. A comparison of prior hand-centric haptic systems and Wireality.

serial communication ( $1 \mathrm{~ms})$, solenoid actuation $(4 \mathrm{~ms})$, ratchet-pawl interfacing $(1 \mathrm{~ms})$, and play in the shoulder mount $(\sim 14 \mathrm{~ms})$. It should be possible to take advantage of actuation lag compensation techniques [8] to reduce latency, as gross arm movements tend to be ballistic.

\section{Field of Reach}

Maximizing freedom of movement is crucial in creating an immersive VR interaction. Our system allows a range of motion with a radius of $83 \mathrm{~cm}$ from the wearer's shoulder, which we found to be sufficient to cover virtual objects over a wide range of distances and angles to the wearer.

\section{Spatial Consistency}

Convincing haptic feedback needs to be spatially accurate able to arrest users' joints at controlled and consistent distances. As a test, we measured the stop point when a participant's finger collided with a static virtual plane at different movement speeds $(30,60$, and $90 \mathrm{~cm} / \mathrm{s})$. We found a Euclidian distance error standard deviation of $1.8 \mathrm{~cm}$.

\section{Comparative Systems}

Table 1 offers a comparison between Wireality and other hand-centric haptic systems. Among the devices that provide collision feedback, Wireality consumes the least amount of power while providing the strongest force feedback. In addition, the weight of Wireality compares favorably. The use of a ratchet gear as the locking mechanism is also unique among these devices. Finally, we note that although our device focuses on providing collision feedback, our system could be combined with prior work enabling other forms of haptic feedback.

\section{QUALITATIVE STUDY}

Our previous evaluations focused on quantifying the physical attributes of our prototype Wireality implementation. Of course, the quality of haptic sensations is subjective, and thus we ran a supplemental qualitative study with 12 participants ( 8 females, mean age 21 ). Six of our participants had no prior VR experience. The study lasted approximately one hour and paid $\$ 10$.

\section{Apparatus \& Conditions}

We asked participants to sit in a chair in the center of a $2 \times 2 \mathrm{~m}^{2}$ open space. The participants wore an Oculus Rift Headset with an attached Leap Motion sensor. We included three haptic conditions in our qualitative study. The first was bare hands (i.e., no augmentation), which acts as our first baseline. As an additional baseline, we included a controller condition with vibration feedback on hand collision (eccentric rotating mass). Our third and final condition is Wireality, using our seven-string implementation worn on the right shoulder. In all conditions, participants wore a noise-canceling headset to mitigate aural cues as a confounding factor.

\section{Procedure}

In each haptic condition, participants interacted with five exemplary objects: a wall, a $45^{\circ}$ tilted flat surface, a sphere, a pole, and an irregular object (Figure 6). The order of the haptic conditions was randomized, and for each user, the presentation order of the objects was also randomized. Participants were allowed to interact with objects as long as they wished and encouraged to think aloud, with comments recorded by the experimenters. Additionally, participants filled out a brief questionnaire after completing all objects in a condition, which contained three questions on a sevenpoint Likert scale. Specifically, the questions were: How realistic was the feeling of the object? ("1 - extremely unrealistic" to "7 - extremely realistic"); How comfortable was the interaction? ("1 - very uncomfortable" to "7 - very comfortable"). How free was your movement? ("1 - blocked" to "7 - full range of motion").

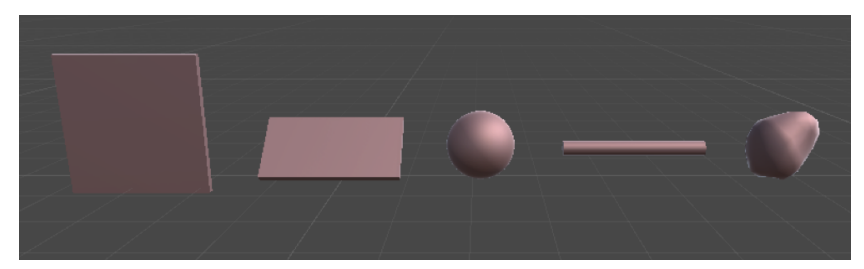

Figure 6. Exemplary objects used in the study. Left to right: a wall, tilted flat surface, sphere, pole, and irregular object. 


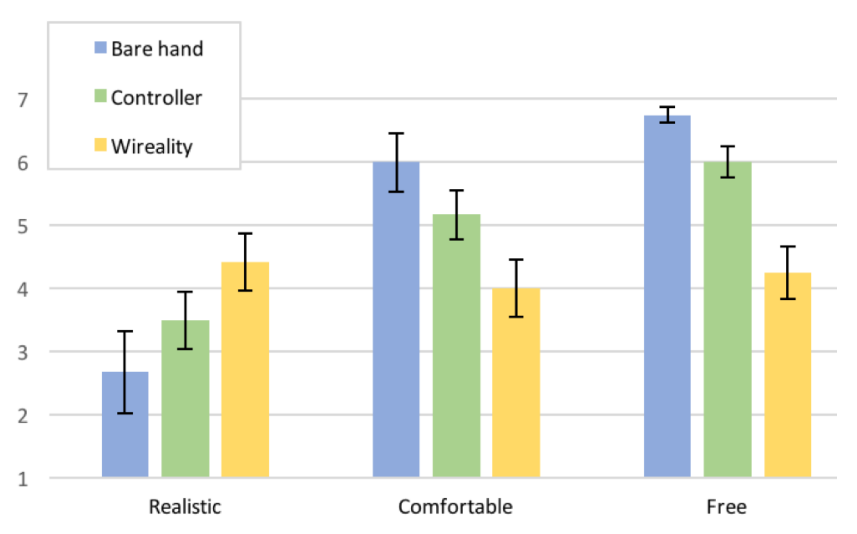

\section{Results}

Figure 7. Questionnaire responses.

Figure 7 shows our main results. We ran a non-parametric Friedman test on our three Likert scales. There was a statistically significant difference in the realistic rating $\left(\chi^{2}(2)=\right.$ 9.591, $\mathrm{p}<.009$ ), and so we performed a post-hoc Wilcoxon signed rank test. We found that Wireality performed significantly better than the bare-hand condition $(p<.05)$ in terms of realism. We also found a significant main effect on comfort $\left(\chi^{2}(2)=12.350, \mathrm{p}<.001\right)$ and freedom of movement $\left(\chi^{2}(2)=17.231, \mathrm{p}<.001\right)$, with post-hoc tests showing that Wireality is significantly worse in these categories verses bare-hand and controller conditions $(p<.006, p<.048$, respectively). There was no significant difference between controller and bare-hand $(\mathrm{p}=.179)$ in terms of comfort, and all conditions were significantly different in freedom of movement $(\mathrm{p}<.05)$.

While we were disappointed that Wireality was rated significantly lower in terms of comfort and freedom of movement, it was not surprising. Compared to bare hands and a small handheld controller, our Wireality prototype is clearly more restrictive and less comfortable. That said, in this research, we focused our engineering efforts on achieving a new level of realism, which our results demonstrate. A commercial implementation would no doubt place greater emphasis on ergonomics.
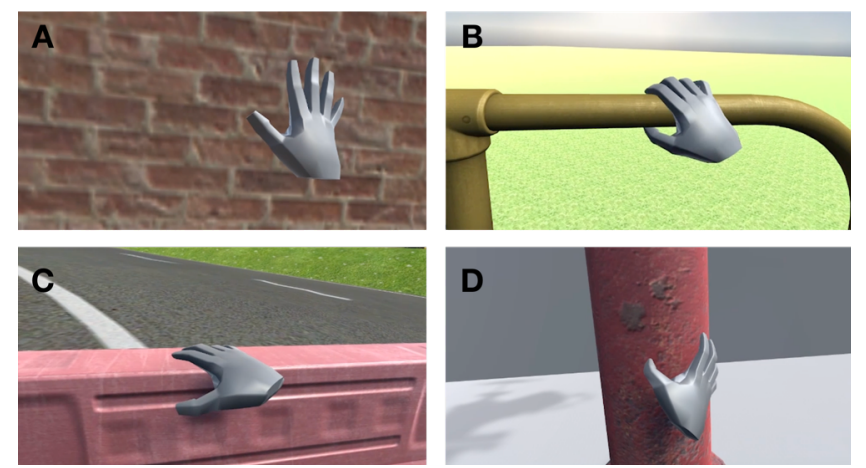

Figure 8. Our system restrains a user's hand collided with a wall (A), railings (B), a roadblock (C), and a fire hydrant (D).

\section{User Feedback}

Overall, participants preferred Wireality over the two baseline conditions for providing a realistic feeling of virtual objects. Participants thought Wireality was a "very intuitive approach" and "closed the feedback loop" of "feeling" virtual objects. Specifically, participants liked the "individualized feedback" given to different parts of the hand as well as having multiple points of haptic feedback in one interaction, especially compared to having no haptic feedback at all or having vibration as the only sensation. Even though participants felt that the controller with vibration feedback was the most accurate in terms of describing the contours of shapes, they noted it did not provide realistic sensations, preferring instead the ability to conform their hands to the shape of objects via Wireality. Participants also reported that during the bare-hand condition, they had to "rely heavily on visual feedback".

In terms of applications, participants thought Wireality could be especially useful in enhancing VR gaming and museum exploration. There were also areas for improvement. Some participants expected their fingers to be pushed from the front instead of being pulled backward. Some also expected to feel the sharpness of edges but were not able to, given the limitations of our device.

\section{EXAMPLE USES}

Based on insights drawn from our user study, we developed a variety of VR scenarios that we believe illustrates the potential of Wireality. These functional examples fall into one of four categories - boundaries, large and heavy objects, interactors, and virtual characters.

\section{Walls, Boundaries \& Infrastructure}

Walls and infrastructure often define the boundaries of environments and thus are essential elements in VR scene design. However, in today's VR systems, hands can simply pass through these obstacles, breaking immersion. In contrast, Wireality can arrest the arm upon contact with boundaries, such as walls and roadblocks, as well as fixed infrastructure, such as railings and fire hydrants (Figure 8, A-D).
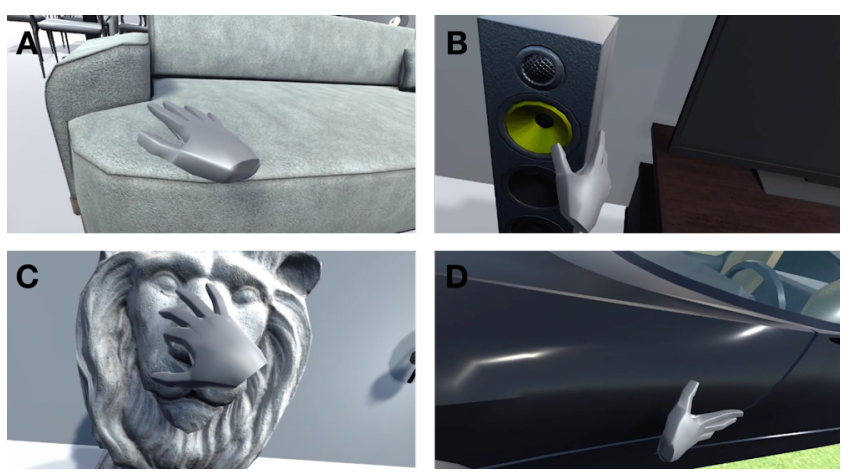

Figure 9. Our system provides force feedback when touching a virtual sofa (A), stereo (B), sculpture (C), and car (D). 

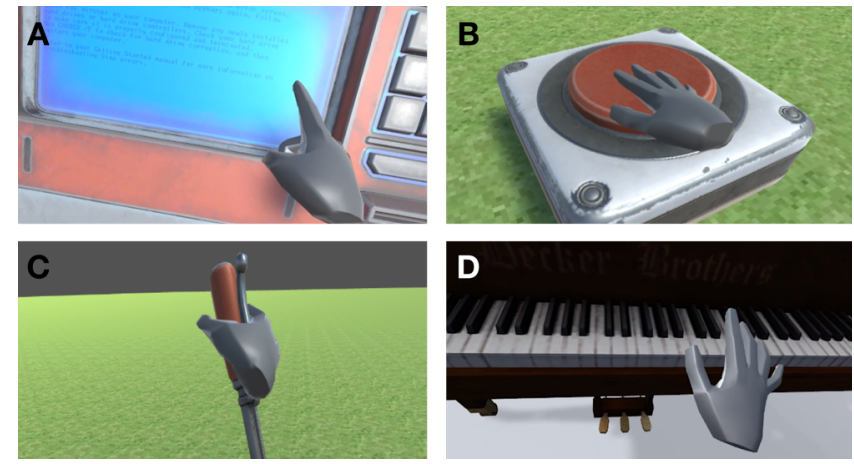

Figure 10. Our system provides force feedback when a user's hand interacts with an ATM touchscreen (A), a button (B), a lever (C), and a piano (D).

\section{Large \& Heavy Objects}

Large and heavy objects, such as furniture and sculptures, also require strong arresting forces. They also tend to have more complex shapes, with corners and edges, which require the user's hand to stop at different points in order to provide a realistic haptic interaction. For demonstration, we built example scenes with four virtual objects: a sofa, a stereo, a sculpture, and a car (Figure 9, A-D).

\section{Interactors}

Wireality can also provide haptic feedback for virtual interactors such as screens and buttons. Figure 10 shows four example scenarios: an ATM screen, a button, a lever, and a piano. Note that some interactors would benefit from more dynamic force rendering (e.g., compliant or multi-stage mechanisms, like levers and press button), which our current system cannot provide.

\section{Characters}

Finally, simulating physical interactions between users and virtual characters (e.g., NPCs, telepresence avatars) could be valuable. Though our system can only provide forces in one direction, we found it sufficient to render a wide range of haptic sensations, such as a high five, tap on the shoulder, shaking hands, and slapping a face (Figure 11, A-D).

\section{LIMITATIONS \& FUTURE WORK}

Wireality makes important tradeoffs in order to achieve its design objectives. Most notable among these is the inability to provide resistive force in any other direction than perpendicular to the wearer's body. This is not as severe in practice as it might seem, as users generally approach objects in VR in a frontal fashion. Even reaching out towards an angled surface feels realistic, as the perpendicular force feels akin to surface friction.

Wireality's simulation of flat surfaces fails when a user translates their hands after touching a surface or object. Once the ratchets are locked and the strings are in tension, the movement would then be constrained to a roughly spherical domain. This too may seem catastrophic, but we found that at arm's length, the arc of a hand feels very much like a flat wall, especially when given strong visual
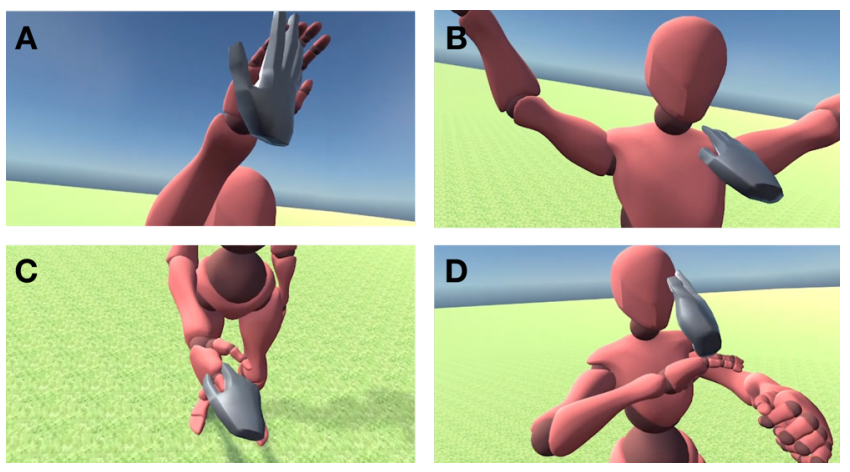

Figure 11. Our system provides haptic feedback when a user's hand interacts with virtual characters: high five (A), tap on shoulder (B), shaking hands (C), and face slapping (D).

cues. However, the illusion breaks for more complex geometries, such as objects with corners or irregular surfaces. We suspect that visuo-haptic illusions (e.g., haptic retargeting) could be used to great effect to mitigate this limitation, a topic we leave for future work.

This inability for a latch to be released without withdrawing one's hands also means users cannot explore complex geometries in a continuous forward motion, but rather requires "pawing" at objects. This limitation also means Wireality cannot fully simulate e.g., buttons and switches, with two-stage interactions. To overcome this limitation, we could provide visual feedback to encourage users to retract their hands. For example, we could illustrate handprints or change hand appearance when encountering a surface, but not during gliding motions, to encourage users to withdraw their hand between interactions.

Another limitation is the inability to simulate the grasping of smaller objects, like a pen or a coffee mug. Any geometry that involves the fingers curling, such that there is no "line of sight" (i.e., uninterrupted air path) between the hand attachment and the corresponding haptic module, means the string will press against the skin, reducing realism by adding an unrelated sensation. Despite this, we can still exert forces on the fingertips, though the illusion is much less convincing than that of larger objects. Another force we cannot simulate is the mass of handheld objects this is again why we focus on large, stationary objects that are placed on the ground (e.g., furniture) and fixed obstacles (e.g., walls) - things that one does not pick up.

We also note that while the retraction force of a single module is not particularly strong $(80 \mathrm{~g})$, the cumulative effect of many modules pulling on the hand is noticeable (e.g., 560g in our proof-of-concept seven-module implementation). However, future module designs could utilize less pull force. Finally, we note that Wireality could be combined with other haptic systems and feedback types to overcome limitations and enable an even greater fidelity of haptic feedback in future VR systems. 


\section{CONCLUSION}

We have described our work on Wireality, a string-based haptic system for virtual reality that enables physical interactions on a wide variety of complex surfaces in a form factor that can be worn and battery-powered. Users wearing our device can physically interact with obstacles and large items, such as walls, furniture, and even virtual characters. These types of elements are key to building immersive virtual worlds, and yet contemporary VR systems do little more than vibrate hand controllers. We show through performance evaluations that our system is both fast and precise, while our qualitative user studies demonstrate how our approach is significantly more realistic that contemporary haptic techniques. Our proof-of-concept implementation is compact, lightweight and low cost - key criteria for a consumer VR accessory.

\section{REFERENCES}

[1] Amazon.com. Aideepen 1.5A L298N 2-Way DC Motor Driver Module. Last Retrieved September 19, 2019 from: https://amazon.com/dp/B075S368Y2

[2] Bruno Araujo, Ricardo Jota, Varun Perumal, Jia Xian Yao, Karan Singh, and Daniel Wigdor. 2016. Snake Charmer: Physically Enabling Virtual Objects. In Proceedings of the TEI '16: Tenth International Conference on Tangible, Embedded, and Embodied Interaction (TEI '16). ACM, New York, NY, USA, 218-226. DOI: https://doi.org/10.1145/2839462.2839484

[3] Paolo Arcara, Luigi Di Stefano, Stefano Mattoccia, Claudio Melchiorri, and Gabriele Vassura. "Perception of depth information by means of a wire-actuated haptic interface." In Proceedings 2000 ICRA. Millennium Conference. IEEE International Conference on Robotics and Automation. Symposia Proceedings (Cat. No. 00CH37065), vol. 4, pp. 3443-3448. IEEE, 2000.

[4] Paolo Arcara, and Claudio Melchiorri. "3D position measurement based on force sensors for a one-wire haptic interface." In Proceedings of the 16th IEEE Instrumentation and Measurement Technology Conference (IMTC '99), Cat. No. 99CH36309, vol. 2, pp. 1272-1277. IEEE, 1999.

[5] Hrvoje Benko, Christian Holz, Mike Sinclair, and Eyal Ofek. 2016. NormalTouch and TextureTouch: Highfidelity 3D Haptic Shape Rendering on Handheld Virtual Reality Controllers. In Proceedings of the 29th Annual Symposium on User Interface Software and Technology (UIST '16). ACM, New York, NY, USA, 717-728. DOI: https://doi.org/10.1145/2984511.2984526

[6] C. Bonivento, A. Eusebi, C. Melchiorri, M. Montanari, and G. Vassura. "WireMan: A portable wire manipulator for touch-rendering of bas-relief virtual surfaces." In 1997 In Proceedings of 8th International Conference on Advanced Robotics (ICAR'97), pp. 13-18. IEEE, 1997.
[7] Daniel K.Y. Chen, Jean-Baptiste Chossat, and Peter B. Shull. 2019. HaptiVec: Presenting Haptic Feedback Vectors in Handheld Controllers using Embedded Tactile Pin Arrays. In Proceedings of the 2019 CHI Conference on Human Factors in Computing Systems (CHI '19). ACM, New York, NY, USA, Paper 171, 11 pages. DOI: https://doi.org/10.1145/3290605.3300401

[8] Inrak Choi, Elliot W. Hawkes, David L. Christensen, Christopher J. Ploch, and Sean Follmer. "Wolverine: A wearable haptic interface for grasping in virtual reality." In 2016 IEEE/RSJ International Conference on Intelligent Robots and Systems (IROS), pp. 986-993. IEEE, 2016.

[9] Inrak Choi, Heather Culbertson, Mark R. Miller, Alex Olwal, and Sean Follmer. 2017. Grabity: A Wearable Haptic Interface for Simulating Weight and Grasping in Virtual Reality. In Proceedings of the 30th Annual ACM Symposium on User Interface Software and Technology (UIST '17). ACM, New York, NY, USA, 119-130. DOI:

https://doi.org/10.1145/3126594.3126599

[10] Inrak Choi, Eyal Ofek, Hrvoje Benko, Mike Sinclair, and Christian Holz. 2018. CLAW: A Multifunctional Handheld Haptic Controller for Grasping, Touching, and Triggering in Virtual Reality. In Proceedings of the 2018 CHI Conference on Human Factors in Computing Systems (CHI '18). ACM, New York, NY, USA, Paper 654, 13 pages. DOI: https://doi.org/10.1145/3173574.3174228

[11] CyberTouch2. Retrieved September 14, 2019 from: http://www.cyberglovesystems.com/cybertouch2/

[12] Alexandra Delazio, Ken Nakagaki, Roberta L. Klatzky, Scott E. Hudson, Jill Fain Lehman, and Alanson P. Sample. 2018. Force Jacket: Pneumatically-Actuated Jacket for Embodied Haptic Experiences. In Proceedings of the 2018 CHI Conference on Human Factors in Computing Systems (CHI '18). ACM, New York, NY, USA, Paper 320, 12 pages. DOI: https://doi.org/10.1145/3173574.3173894

[13] 12V DC suction micro electromagnet. Last Retrieved September 19, 2019 from: https://ebay.com/itm/12VDC-suction-micro-electromagnet-spring-push-pulltype-rod-solenoid-magnet-VvV/123830128835

[14] Facebook Inc. Oculus Rift. Last Retrieved September 16, 2019. from: https://www.oculus.com/rift

[15] Facebook Inc. Oculus Quest. Last Retrieved September 19, 2019. from: https://www.oculus.com/quest

[16] Hossein S. Farahani, and Jeha Ryu. "Design and control of a wire-driven haptic device: Hapticpen." 2005.

[17] Flexguard. RSCC Sub-Mini Retractor. Last Retrieved September 19, 2019 from: https://flexguard.com/collections/retractors/products/rs cc-retractor 
[18] Gemperle, C. Kasabach, J. Stivoric, M. Bauer, and R. Martin. 1998. Design for Wearability. In Proceedings of the 2nd IEEE International Symposium on Wearable Computers (ISWC '98). IEEE Computer Society, Washington, DC, USA, 116-.

[19] Clément Gosselin. "Cable-driven parallel mechanisms: state of the art and perspectives." Mechanical Engineering Reviews, 1, no. 1 (2014): DSM0004DSM0004.

[20] João Guerreiro, Hernisa Kacorri, Jeffrey P. Bigham, Edward Cutrell, Daisuke Sato, Dragan Ahmetovic, and Chieko Asakawa. 2019. Hacking Blind Navigation: "In-Sight: Tension-Based Feedback for Navigation" In Extended Abstracts of the 2019 CHI Conference on Human Factors in Computing Systems (CHI EA '19). ACM, New York, NY, USA, Paper W10, 8 pages. DOI: https://doi.org/10.1145/3290607.3299015

[21] Jan Gugenheimer, Dennis Wolf, Eythor R. Eiriksson, Pattie Maes, and Enrico Rukzio. 2016. GyroVR: Simulating Inertia in Virtual Reality using Head Worn Flywheels. In Proceedings of the 29th Annual Symposium on User Interface Software and Technology (UIST '16). ACM, New York, NY, USA, 227-232. DOI: https://doi.org/10.1145/2984511.2984535

[22] Abhishek Gupta, and Marcia K. O'Malley. "Design of a haptic arm exoskeleton for training and rehabilitation." IEEE/ASME Transactions on mechatronics, 11 , no. 3 (2006): 280-289.

[23] HaptX Inc. VR haptic glove. Retrieved September 14, 2019 from: https://haptx.com

[24] Seongkook Heo, Christina Chung, Geehyuk Lee, and Daniel Wigdor. 2018. Thor's Hammer: An Ungrounded Force Feedback Device Utilizing Propeller-Induced Propulsive Force. In Proceedings of the 2018 CHI Conference on Human Factors in Computing Systems (CHI '18). ACM, New York, NY, USA, Paper 525, 11 pages. DOI: https://doi.org/10.1145/3173574.3174099

[25] Ronan Hinchet, Velko Vechev, Herbert Shea, and Otmar Hilliges. 2018. DextrES: Wearable Haptic Feedback for Grasping in VR via a Thin Form-Factor Electrostatic Brake. In Proceedings of the 31st Annual ACM Symposium on User Interface Software and Technology (UIST '18). ACM, New York, NY, USA, 901-912. DOI:

https://doi.org/10.1145/3242587.3242657

[26] Matthias Hoppe, Pascal Knierim, Thomas Kosch, Markus Funk, Lauren Futami, Stefan Schneegass, Niels Henze, Albrecht Schmidt, and Tonja Machulla. 2018. VRHapticDrones: Providing Haptics in Virtual Reality through Quadcopters. In Proceedings of the 17th International Conference on Mobile and Ubiquitous Multimedia (MUM 2018). ACM, New York, NY, USA, 718. DOI: https://doi.org/10.1145/3282894.3282898
[27]Zhu Hou, Yuru Zhang, and Yi Yang. "Enhancing touch screen games through a cable-driven force feedback device." In 2012 International Conference on Virtual Reality and Visualization, pp. 56-61. IEEE, 2012.

[28] HTC Corporation. Vive. Last Retrieved September 20 2019 from https:/www.vive.com/

[29] Masahiro Ishii and Makoto Sato. 1994. A 3d spatial interface device using tensed strings. Presence: Teleoper. Virtual Environ. 3, 1 (January 1994), 81-86. DOI=http://dx.doi.org/10.1162/pres.1994.3.1.81

[30] Ali Israr and Ivan Poupyrev. 2011. Tactile brush: drawing on skin with a tactile grid display. In Proceedings of the SIGCHI Conference on Human Factors in Computing Systems (CHI '11). ACM, New York, NY, USA, 2019-2028. DOI: https://doi.org/10.1145/1978942.1979235

[31] Saurabh Jadhav, Vikas Kannanda, Bocheng Kang, Michael T. Tolley, and Jurgen P. Schulze. "Soft robotic glove for kinesthetic haptic feedback in virtual reality environments." Electronic Imaging 2017, no. 3 (2017): 19-24.

[32] Leap Motion Inc. Leap Motion. Last Retrieved September 16, 2019 from: https://www.leapmotion.com

[33] Jaeyeon Lee, Mike Sinclair, Mar Gonzalez-Franco, Eyal Ofek, and Christian Holz. 2019. TORC: A Virtual Reality Controller for In-Hand High-Dexterity Finger Interaction. In Proceedings of the 2019 CHI Conference on Human Factors in Computing Systems (CHI '19). ACM, New York, NY, USA, Paper 71, 13 pages. DOI: https://doi.org/10.1145/3290605.3300301

[34] Robert W. Lindeman, Yasuyuki Yanagida, Haruo Noma, and Kenichi Hosaka. "Wearable vibrotactile systems for virtual contact and information display." Virtual Reality, 9, no. 2-3 (2006): 203-213.

[35] Pedro Lopes, Sijing You, Lung-Pan Cheng, Sebastian Marwecki, and Patrick Baudisch. 2017. Providing Haptics to Walls \& Heavy Objects in Virtual Reality by Means of Electrical Muscle Stimulation. In Proceedings of the 2017 CHI Conference on Human Factors in Computing Systems (CHI '17). ACM, New York, NY, USA, 1471-1482. DOI: https://doi.org/10.1145/3025453.3025600

[36] Nuratiqa Natrah Mansor, Muammad Herman Jamaluddin, and Ahamad Zaki Shukor. "Concept and Application of Virtual Reality Haptic Technology: A Review." Journal of Theoretical \& Applied Information Technology, 95, no. 14 (2017).

[37] Markforged, Inc. 800cc Onyx Filament Spool. Last Retrieved September 19, 2019 from https://markforged.com/product/onyx-filament/

[38] Claudio Melchiorri, Gabriele Vassura, and Paolo Arcara. "What kind of haptic perception can we get with a one-wire interface?." In Proceedings 1999 IEEE Inter- 
national Conference on Robotics and Automation (Cat. No. 99CH36288C), vol. 2, pp. 1620-1625. IEEE, 1999.

[39] Victor Adriel de Jesus Oliveira, Luciana Nedel, Anderson Maciel, and Luca Brayda. "Localized magnification in vibrotactile HMDs for accurate spatial awareness." In International Conference on Human Haptic Sensing and Touch Enabled Computer Applications, pp. 55-64. Springer, Cham, 2016.

[40] Martin J-D. Otis, Thien-Ly Nguyen-Dang, Thierry Laliberte, Denis Ouellet, Denis Laurendeau, and Clément Gosselin. "Cable tension control and analysis of reel transparency for 6-dof haptic foot platform on a cable-driven locomotion interface." International Journal of Electrical and Electronics Engineering, 3, no. 1 (2009): 16-29.

[41] Roshan Lalintha Peiris, Wei Peng, Zikun Chen, Liwei Chan, and Kouta Minamizawa. 2017. ThermoVR: Exploring Integrated Thermal Haptic Feedback with Head Mounted Displays. In Proceedings of the 2017 CHI Conference on Human Factors in Computing Systems (CHI '17). ACM, New York, NY, USA, 5452-5456. DOI: https://doi.org/10.1145/3025453.3025824

[42] Usman Sargaana, H. S. Farahani, J. Lee, Jeha Ryu, and W. Woo. "billiARds: Augmented Reality System with Wearable Force-Feedback Device." In International Conference on Human Computer Interaction. 2005.

[43] Henning Schmidt. "HapticWalker-A novel haptic device for walking simulation." In Proceedings of EuroHaptics, vol. 2, pp. 60-67. 2004.

[44] Erik H. Skorina, Ming Luo, and Cagdas D. Onal. "A soft robotic wearable wrist device for kinesthetic haptic feedback." Frontiers in Robotics and AI, 5 (2018): 83.

[45] Kahye Song, Sung Hee Kim, Sungho Jin, Sohyun Kim, Sunho Lee, Jun-Sik Kim, Jung-Min Park, and Youngsu Cha. "Pneumatic actuator and flexible piezoelectric sensor for soft virtual reality glove system." Scientific reports, 9, no. 1 (2019): 8988.

[46] Evan Strasnick, Christian Holz, Eyal Ofek, Mike Sinclair, and Hrvoje Benko. 2018. Haptic Links: Bimanual Haptics for Virtual Reality Using Variable Stiffness Actuation. In Proceedings of the 2018 CHI Conference on Human Factors in Computing Systems (CHI '18). ACM, New York, NY, USA, Paper 644, 12 pages. DOI: https://doi.org/10.1145/3173574.3174218

[47] Yuqian Sun, Shigeo Yoshida, Takuji Narumi, and Michitaka Hirose. 2019. PaCaPa: A Handheld VR Device for Rendering Size, Shape, and Stiffness of Virtual Objects in Tool-based Interactions. In Proceedings of the 2019 CHI Conference on Human Factors in Computing Systems (CHI '19). ACM, New York, NY, USA, Paper 452, 12 pages. DOI: https://doi.org/10.1145/3290605.3300682
[48] Taclim Inc. VR haptic boot. Retrieved September 14, 2019 from https://taclim.cerevo.com/en/

[49] PJRC Inc. Teensy 3.2. Retrieved September 16, 2019 from: https://www.pjrc.com/store/teensy32.html

[50] Shan-Yuan Teng, Tzu-Sheng Kuo, Chi Wang, Chihuan Chiang, Da-Yuan Huang, Liwei Chan, and BingYu Chen. 2018. PuPoP: Pop-up Prop on Palm for Virtual Reality. In Proceedings of the 31st Annual ACM Symposium on User Interface Software and Technology (UIST '18). ACM, New York, NY, USA, 5-17. DOI: https://doi.org/10.1145/3242587.3242628

[51] Hsin-Ruey Tsai, Jun Rekimoto, and Bing-Yu Chen. 2019. ElasticVR: Providing Multilevel ContinuouslyChanging Resistive Force and Instant Impact Using Elasticity for VR. In Proceedings of the 2019 CHI Conference on Human Factors in Computing Systems (CHI '19). ACM, New York, NY, USA, 220. DOI: https://doi.org/10.1145/3290605.3300450

[52] VRGluv Inc. VR haptic glove. Retrieved September 14, 2019 from https://www.vrgluv.com/technology

[53] Eric Whitmire, Hrvoje Benko, Christian Holz, Eyal Ofek, and Mike Sinclair. 2018. Haptic Revolver: Touch, Shear, Texture, and Shape Rendering on a Reconfigurable Virtual Reality Controller. In Proceedings of the 2018 CHI Conference on Human Factors in Computing Systems (CHI '18). ACM, New York, NY, USA, Paper 86, 12 pages. DOI:

https://doi.org/10.1145/3173574.3173660

[54] Robert L. Williams II "Cable-suspended haptic interface." International Journal of Virtual Reality, 3, no. 3 (1998): 13-21.

[55] Yi Yang, and Yuru Zhang. "A new cable-driven haptic device for integrating kinesthetic and cutaneous display." In 2009 ASME/IFToMM International Conference on Reconfigurable Mechanisms and Robots, pp. 386-391. IEEE, 2009.

[56] Yuhang Zhao, Cynthia L. Bennett, Hrvoje Benko, Edward Cutrell, Christian Holz, Meredith Ringel Morris, and Mike Sinclair. 2018. Enabling People with Visual Impairments to Navigate Virtual Reality with a Haptic and Auditory Cane Simulation. In Proceedings of the 2018 CHI Conference on Human Factors in Computing Systems (CHI '18). ACM, New York, NY, USA, Paper 116, 14 pages. DOI: https://doi.org/10.1145/3173574.3173690

[57] Ma Zhou, and Pinhas Ben-Tzvi. "RML glove-An exoskeleton glove mechanism with haptics feedback." IEEE/ASME Transactions on Mechatronics, 20, no. 2 (2014): 641-652. 